\title{
An evolutive discrete exchange economy model with heterogeneous preferences
}

\author{
Ahmad Naimzada a1 , Marina Pireddu b2 \\ ${ }^{a}$ Dept. of Economics, Management and Statistics, University of Milano-Bicocca, \\ U6 Building, Piazza dell'Ateneo Nuovo 1, 20126 Milano, Italy. \\ ${ }^{\mathrm{b}}$ Dept. of Mathematics and Applications, University of Milano-Bicocca, \\ U5 Building, Via Cozzi 55, 20125 Milano, Italy.
}

\begin{abstract}
We propose an exchange economy evolutionary model with discrete time, in which there are two groups of agents characterized by different structures of preferences. The share updating mechanism depends in a monotone manner on the goods' consumption, described in terms of the calorie intakes. In such framework we investigate the existence of equilibria, their stability and the occurrence of multistability phenomena via a qualitative bifurcation analysis, which also highlights the presence of transcritical bifurcations.

Keywords: Endogenous preferences, evolution, coexistence, bifurcation, complex dynamics, multistability
\end{abstract}

\section{Introduction}

In the present paper we are going to show the richness of dynamic behaviors arising from the exchange economy evolutionary model introduced in [1] when considering time as discrete rather than continuous. Indeed, like in that paper we deal with an exchange economy setting in which agents are heterogeneous in the structure of preferences. Namely, the weights assigned to the two consumption goods in the Cobb-Douglas utility functions do not coincide across

\footnotetext{
${ }^{1}$ Tel.: +39 0264485813; fax: +39 0264483085. E-mail address: ahmad.naimzada@unimib.it

${ }^{2}$ Corresponding author. Tel.: +390264485767; fax: +390264485705. E-mail address: marina.pireddu@unimib.it
} 
groups. Moreover, similarly to [1], the mechanism according to which shares are updated depends on goods' consumption, described in terms of the calorie intakes of the two groups of agents. However, instead of the linear, monotone dependence considered therein, we assume a nonlinear, but still monotone, relationship between the share updating mechanism and the (difference of the) calorie intakes. In particular, following [11, 12], we do consider an exponential discrete replicator rule to describe the evolutionary mechanism. We recall that, according to [1], a monotone population growth rate is suitable to represent the long-run centuries-old trend, as the diet of a population group affects its long-term survival. More precisely, as observed in [8], biological payoff functions monotonically increasing in the calorie intake well describe food regimes characterized by a calorie shortage, and are thus appropriate to represent the long-run centuries-old trend before the industrial revolution.

The model we propose belongs to the line of research developed in $[7,8,9]$ and inspired to the setting in [1]. In more detail, in [7, 8] time is continuous and the focus is on the analysis of the local stability of the equilibria and on some of their static features, such as weak and strong coexistence between groups, assuming that endowments are respectively homogeneous and heterogeneous between groups. In those papers we replaced the monotone population growth rate assumed in [1] with a bell-shaped map, increasing with the calorie intake up to a certain threshold value, above which it becomes decreasing. Bellshaped maps are indeed well-suited to describe the framework of contemporary developed countries and the negative effects of overconsumption on health and survival (see $[7,10])$. In $[9]$ the evolutionary mechanism is based on the relative utility values realized by the two kinds of agents, rather than on biological payoffs.

As mentioned above, differently from [1], in the present paper we do consider time as discrete, rather than continuous. However, we stress that we do not deal with a numerical discretization of the model proposed therein. Indeed, our discretization is built on the assumptions we make on the evolutionary mechanism. In particular, we describe it via an exponential replicator rule (see $[11,12])$. The 
choice of dealing with a discrete-time, rather than continuous-time, model comes from the consideration that the former framework is more suitable to represent the sequence of actions which lead to the formation of the population shares. Namely, in view of embracing a new preference structure, agents need time to evaluate the satisfaction degree resulting from their previous choice, to gather information on the other lifestyles and to compare the various satisfaction levels, in order to make their next choice.

We find that the passage from continuous to discrete time is not innocuous in terms of results. More precisely, like in [1], in addition to the two trivial market stationary equilibria, in which just one of the two groups of agents is present, we find at most one nontrivial equilibrium, characterized by the coexistence between the two groups. We perform a qualitative bifurcation analysis on varying mainly the parameter measuring the heterogeneity in the structure of preferences between groups and we prove that the nontrivial equilibrium may emerge via a transcritical bifurcation. Thanks to our nonlinearity, such internal equilibrium can be stable or it can lose stability via a flip bifurcation, leading to the presence of oscillatory and chaotic orbits, while in [1] it is always stable. We stress that when it is locally stable, it may be surrounded by periodic or chaotic attractors, due to the presence of multistability phenomena.

The remainder of the paper is organized as follows. In Section 2 we present our model and we analyze the existence and local stability of the equilibria. In Section 3 we perform a qualitative bifurcation analysis, showing the possible dynamic behaviors for the system. In Section 4 we briefly discuss our results and describe possible future study directions.

\section{The model}

We start our discussion recalling the framework in [1], where the authors consider a continuous-time model describing an exchange economy with a continuum of agents, which may be of type $\alpha$ or of type $\beta$. There are two consumption goods, $x$ and $y$, and agent preferences are described by Cobb-Douglas utility functions, i.e., $U_{i}(x, y)=x^{i} y^{1-i}$, for $i \in\{\alpha, \beta\}$, with $0<\beta<\alpha<1$. The 
quantity of good $x(y)$ consumed by an agent of type $i \in\{\alpha, \beta\}$ is denoted by $x_{i}$ $\left(y_{i}\right)$. Both kinds of agents have the same endowments of the two goods, denoted respectively by $w_{x}$ and $w_{y}$. The analysis is performed in terms of the relative price $p(t)=p_{y}(t) / p_{x}(t)$, where $p_{x}(t)$ and $p_{y}(t)$ are the prices at time $t$ for goods $x$ and $y$, respectively. The size of the population of kind $\alpha(\beta)$ at time $t$ is denoted by $A(t)(B(t))$ and the normalized variable $a(t)=A(t) /(A(t)+B(t))$ represents the population fraction composed by the agents of type $\alpha$. For simplicity, we assume that the population size is normalized to 1 , so that the fraction composed by the agents of type $\beta$ is given by $1-a(t)$.

We now present the definition of market equilibrium, we will refer to in the remainder of the paper. With this respect, we stress that the only difference between the framework we are going to consider and the one in [1] is that, in order to take into account the complexity of the evolutive process of share formation and the time it requires, we assume that in our model time is discrete rather than continuous.

Definition 2.1. Given the economy and the population share a(t), a market equilibrium at time $t$ is a vector $\left(p^{*}(t), x_{i}^{*}(t), y_{i}^{*}(t)\right)$, with $i \in\{\alpha, \beta\}$, such that:

- every kind of agent chooses a utility-maximizing consumption bundle, given $p^{*}(t) ;$

- the markets for the two goods clear.

Simple computations show that, solving the consumer maximization problems for agents of type $\alpha$ and $\beta$ and using a market clearing condition, the market equilibrium price is given by

$$
p^{*}(t)=\frac{[1-(a(t) \alpha+(1-a(t)) \beta)] w_{x}}{(a(t) \alpha+(1-a(t)) \beta) w_{y}}
$$

and the consumer equilibrium quantities of the two goods for an agent of type $i \in\{\alpha, \beta\}$ are

$$
\begin{aligned}
& x_{i}^{*}(t)=i\left(w_{x}+p^{*}(t) w_{y}\right)=\frac{i w_{x}}{a(t) \alpha+(1-a(t)) \beta}, \\
& y_{i}^{*}(t)=(1-i)\left(\frac{w_{x}}{p^{*}(t)}+w_{y}\right)=\frac{(1-i) w_{y}}{1-(a(t) \alpha+(1-a(t)) \beta)} .
\end{aligned}
$$


See $[1,7]$ for further mathematical details.

Once we specify a dynamical rule for the population share evolution, it is also possible to give the definition of market stationary equilibrium as follows.

Definition 2.2. Given the economy, the vector $\left(a^{*}, p^{*}, x_{i}^{*}, y_{i}^{*}\right), i \in\{\alpha, \beta\}$, is a market stationary equilibrium if $a^{*}$ is constant and if, given $a^{*},\left(p^{*}, x_{i}^{*}, y_{i}^{*}\right)$, $i \in\{\alpha, \beta\}$, is a market equilibrium for every $t$.

For the sake of brevity, we shall identify market stationary equilibria just with the population share $a$, since it determines all other equilibrium components. The market stationary equilibria, at which for every $t$ the population shares, and thus also the market equilibrium price and the consumer equilibrium quantities, are constant, will be called trivial if they are not characterized by the coexistence between the two groups of agents, and nontrivial otherwise.

Let us now recall the dynamical rule for the population share evolution considered in [1] and based on a biological payoff.

The calorie intake $K_{i}(t)$ of an agent of type $i \in\{\alpha, \beta\}$ at time $t$ is given by a linear combination of the units $x_{i}(t)$ and $y_{i}(t)$ of goods $x$ and $y$ he consumes, weighted respectively with the calories that each agent derives from the consumption of a unit of good $x$ and of good $y$, i.e., $K_{i}(t)=c_{x} x_{i}(t)+c_{y} y_{i}(t)$. Denoting by $\bar{K}$ the calorie subsistence level, in [1] the growth rate of the population of type $i$ is then assumed to be

$$
K_{i}(t)-\bar{K}
$$

so that the evolution of the two groups of consumers is described by the following system

$$
\left\{\begin{array}{l}
\frac{d A(t)}{d t}=\left(K_{\alpha}(t)-\bar{K}\right) A(t) \\
\frac{d B(t)}{d t}=\left(K_{\beta}(t)-\bar{K}\right) B(t)
\end{array}\right.
$$

or equivalently, in terms of the normalized variable $a(t)$, by

$$
\frac{d a(t)}{d t}=a(t)(1-a(t))\left(K_{\alpha}(t)-K_{\beta}(t)\right) .
$$


Hence, since for $i \in\{\alpha, \beta\}$

$$
K_{i}(t)=\frac{i c_{x} w_{x}}{a(t) \alpha+(1-a(t)) \beta}+\frac{(1-i) c_{y} w_{y}}{1-a(t) \alpha-(1-a(t)) \beta},
$$

(2.5) can be rewritten as

$$
\frac{d a(t)}{d t}=(\alpha-\beta) a(t)(1-a(t))\left(\frac{c_{x} w_{x}}{a(t) \alpha+(1-a(t)) \beta}-\frac{c_{y} w_{y}}{1-a(t) \alpha-(1-a(t)) \beta}\right) .
$$

Notice that $\bar{K}$ does not affect the one-dimensional differential equation.

In addition to the trivial market stationary equilibria $a=0$ and $a=1$, a nontrivial market stationary equilibrium is given by $a=a^{*}$, with

$$
a^{*}=\frac{(1-\beta) c_{x} w_{x}-\beta c_{y} w_{y}}{(\alpha-\beta)\left(c_{x} w_{x}+c_{y} w_{y}\right)},
$$

as long as $a^{*} \in(0,1)$, i.e., for $c_{x} w_{x} \in\left(\left(\beta c_{y} w_{y}\right) /(1-\beta),\left(\alpha c_{y} w_{y}\right) /(1-\alpha)\right)$. Such market stationary equilibrium, when it exists, is always stable for the model considered in [1]. In that paper no comments are made on the local stability of the dynamical system at $a=0$ and $a=1$. However, a simple continuity argument shows that, when $a^{*} \in(0,1)$, then $a=0$ and $a=1$ are always unstable. When instead $a^{*} \notin(0,1), a=0$ may be unstable and $a=1$ stable, or vice versa. We stress that, by construction, at $a=a^{*}$ we necessarily have $K_{\alpha}(t)=K_{\beta}(t)$, for every $t$.

As mentioned above, the framework we are going to analyze differs from the one just described as we will take time as discrete, rather than continuous. However, we will not deal with a numerical discretization of (2.5) but, following $[11,12]$, we do consider an exponential discrete replicator mechanism, so that the evolution of the fraction $a(t)$ of traders of type $\alpha$ is described by the discrete choice model

$$
a(t+1)=\frac{a(t) \exp \left(\mu\left(K_{\alpha}(t)-\bar{K}\right)\right)}{a(t) \exp \left(\mu\left(K_{\alpha}(t)-\bar{K}\right)\right)+(1-a(t)) \exp \left(\mu\left(K_{\beta}(t)-\bar{K}\right)\right)},
$$

where $\mu$ is a positive parameter describing the intensity of the reactivity to changes in the calorie intakes for the two groups of agents. We stress that our choice does not alter, with respect to the setting in [1], the market equilibrium 
price and the consumer equilibrium quantities, which are still represented by (2.1) and (2.2), respectively. Hence, also the expressions for $K_{\alpha}(t)$ and $K_{\beta}(t)$ in (2.6) remain unchanged.

Notice that, performing simple algebraic operations, (2.9) can be rewritten as

$$
a(t+1)=\frac{a(t)}{a(t)+(1-a(t)) \exp \left(\mu\left(K_{\beta}(t)-K_{\alpha}(t)\right)\right)} .
$$

We remark that, although (2.10) expresses a nonlinear relation between $K_{\alpha}(t)-$ $K_{\beta}(t)$ and $a(t+1)$, the latter is increasing in $K_{\alpha}(t)-K_{\beta}(t)$, just like the linear relation in (2.5). Moreover, as happens with (2.5), in our model the parameter $\bar{K}$ does not influence the dynamics. Furthermore, like we shall see in Proposition 2.1 below, when looking for the market stationary equilibria, we find exactly the same steady states as in [1], i.e., $a=0, a=1$ and $a=a^{*}$ in (2.8).

As concerns parameter $\mu$, in the limit $\mu \rightarrow 0(2.10)$ simply reads as $a(t+1)=$ $a(t), \forall t$, so that initial conditions are maintained and agents are insensitive to the values of the calorie intakes $K_{\alpha}$ and $K_{\beta}$. At the other extreme, when $\mu \rightarrow$ $+\infty$, the population switches are fully governed by the rational component and all agents instantaneously move towards the preference structure characterized by a larger calorie intake. Namely, if $K_{\alpha}<K_{\beta}$, then for $\mu \rightarrow+\infty$ we have $a(t+1) \rightarrow 0$, while if $K_{\alpha}>K_{\beta}$, then $a(t+1) \rightarrow 1$.

In view of the subsequent analysis, it is expedient to introduce the onedimensional map $f:[0,1] \rightarrow \mathbb{R}$ related to (2.9), and defined as

$$
f(a)=\frac{a}{a+(1-a) \exp \left(\mu\left(K_{\beta}-K_{\alpha}\right)\right)},
$$

with $K_{\alpha}$ and $K_{\beta}$ as in (2.6).

As a first step in the study of our dynamical system, in the next result we derive the expressions of the market stationary equilibria for (2.9).

Proposition 2.1. Equation (2.9) admits as market stationary equilibria $a=$ $0, a=1$ and $a=a^{*}$ in (2.8), as long as

$$
\frac{c_{x} w_{x}}{c_{y} w_{y}} \in\left(\frac{\beta}{1-\beta}, \frac{\alpha}{1-\alpha}\right) .
$$


Proof. The conclusion immediately follows by observing that $a=0, a=1$ and $a=a^{*}$ in (2.8) are all the solutions to the fixed-point equation $f(a)=a$, with $f$ as in (2.11). Condition (2.12) is equivalent to $a^{*} \in(0,1)$.

Notice that, introducing the heterogeneity degree between groups $\Delta=\alpha-\beta$, measuring the difference in the preference structures, it is possible to rewrite (2.12) as

$$
\frac{c_{x} w_{x}}{c_{y} w_{y}} \in\left(\frac{\beta}{1-\beta}, \frac{\beta+\Delta}{1-\beta-\Delta}\right) .
$$

Since the lower bound does not depend on $\Delta$ and the upper bound $\frac{\beta+\Delta}{1-\beta-\Delta}=$ $\frac{1}{1-\beta-\Delta}-1$ is increasing in $\Delta$, we can infer that for large values of the heterogeneity degree it becomes easier to have $a^{*} \in(0,1)$. Moreover, rewriting $a^{*}$ in (2.8) in terms of $\Delta$ as

$$
a^{*}=\frac{(1-\beta) c_{x} w_{x}-\beta c_{y} w_{y}}{\Delta\left(c_{x} w_{x}+c_{y} w_{y}\right)},
$$

it becomes evident that $a^{*}$ is decreasing in $\Delta$, as long as it is positive. We find a confirmation of this in the bifurcation diagrams in Figures 1 and 3. It is also clear that $a^{*}$ is decreasing in $c_{y}$, too, as confirmed by Figures 7-9.

We can explain such decreasing behavior of $a^{*}$ both with respect to $\Delta$ and $c_{y}$ as follows.

When $\Delta$ grows, since $\beta$ is fixed and $\alpha=\beta+\Delta$ increases, the preference of the agents of type $\alpha$ for commodity $x$ becomes stronger. Hence, the individual demand of the agents of type $\alpha$ for commodity $x$ raises, and consequently also the aggregate demand for commodity $x$ raises. This makes the corresponding price $p_{x}$ increase, with a resulting decrease in the relative price $p=p_{y} / p_{x}$. The market closes and the optimal consumption quantities for the two goods are determined. Due to the increase in $p_{x}$, agents of type $\alpha$ consume a lower amount of commodity $x$ and a decrease in $x_{\alpha}^{*}$ induces a lower calorie intake for the agents of type $\alpha$, so that their share decreases.

As concerns the decrease of $a=a^{*}$ with respect to $c_{y}$, we notice that an increase in $c_{y}$ favors the agents of group $\beta$, because of their stronger preference for commodity $y$, and this leads to a decrease in the share of agents of group $\alpha$. 
In the next result, we report the stability conditions at the three equilibria.

Proposition 2.2. Equation (2.9) is locally asymptotically stable:

- at 0 if $\frac{c_{x} w_{x}}{c_{y} w_{y}}<\frac{\beta}{1-\beta}$;

- at $a^{*} \in(0,1)$ in $(2.8)$ if $(2.12)$ is satisfied and $\mu<\mu^{*}=\frac{2\left(c_{x} w_{x} c_{y} w_{y}\right)}{\left(c_{x} w_{x}+c_{y} w_{y}\right)\left((\alpha+\beta-2 \alpha \beta) c_{x} w_{x} c_{y} w_{y}-(1-\alpha)(1-\beta) c_{x}^{2} w_{x}^{2}-\alpha \beta c_{y}^{2} w_{y}^{2}\right)} ;$

- at 1 if $\frac{c_{x} w_{x}}{c_{y} w_{y}}>\frac{\alpha}{1-\alpha}$.

In particular, a flip bifurcation occurs at $a=a^{*}$ if $\mu=\mu^{*}$.

Proof. Since $f^{\prime}(0)>-1, f^{\prime}\left(a^{*}\right)<1$ and $f^{\prime}(1)>-1$, with $a^{*} \in(0,1)$ and $f$ as in (2.11), are always fulfilled, the stability conditions follow by imposing respectively $f^{\prime}(0)<1, f^{\prime}\left(a^{*}\right)>-1$ and $f^{\prime}(1)<1$. As concerns the stability conditions at $a=a^{*}$, we remark that simple computations show that the factor $\left((\alpha+\beta-2 \alpha \beta) c_{x} w_{x} c_{y} w_{y}-(1-\alpha)(1-\beta) c_{x}^{2} w_{x}^{2}-\alpha \beta c_{y}^{2} w_{y}^{2}\right)$ is positive when (2.12) is satisfied. The condition for the flip bifurcation follows by setting $f^{\prime}\left(a^{*}\right)=-1$.

We stress that the stability conditions for (2.9) at $a=0$ and $a=1$ coincide with those we computed for the framework considered in [1] and are fulfilled only when $a^{*} \notin(0,1)$. Differently from that context, the stability at $a=a^{*}$ is instead no more granted here and depends on the value of the various parameters. We shall illustrate some possible scenarios in Figures 1-9. In particular, in Figures 1-6 we will consider the heterogeneity degree $\Delta=\alpha-\beta$ as bifurcation parameter. With this respect we notice that the stability condition found in Proposition 2.2 at $a=a^{*}$ may be rewritten in terms of $\Delta$ as stated in the following result.

Corollary 2.1. Equation (2.9) is locally asymptotically stable at $a^{*} \in(0,1)$ in (2.8) if (2.12) is satisfied and 


$$
\Delta<\Delta^{*}=\frac{\frac{2 c_{x} w_{x} c_{y} w_{y}}{\mu\left(c_{x} w_{x}+c_{y} w_{y}\right)}+(1-\beta)^{2} c_{x}^{2} w_{x}^{2}+\beta^{2} c_{y}^{2} w_{y}^{2}-2 \beta(1-\beta) c_{x} w_{x} c_{y} w_{y}}{(1-\beta) c_{x}^{2} w_{x}^{2}-\beta c_{y}^{2} w_{y}^{2}+(1-2 \beta) c_{x} w_{x} c_{y} w_{y}} .
$$

In particular, a flip bifurcation occurs at $a=a^{*}$ if $\Delta=\Delta^{*}$.

Proof. The result immediately follows by Proposition 2.2. We just remark that simple computations show that $(1-\beta) c_{x}^{2} w_{x}^{2}-\beta c_{y}^{2} w_{y}^{2}+(1-2 \beta) c_{x} w_{x} c_{y} w_{y}$ is positive when (2.12) is fulfilled.

In Figures 7-9 as bifurcation parameter we will consider instead $c_{y}$. We observe that the stability condition derived in Proposition 2.2 at $a=a^{*}$, when expanded, contains terms of degree 3 in $c_{y}$ and, thus, finding the stability threshold values in terms of such parameter would require cumbersome computations. Nonetheless, as we will more precisely explain in Section 3, we checked the consistency of the stability threshold values for $c_{y}$ that we inferred from the bifurcation diagrams reported in Figures 8 and 9 with the stability condition at $a=a^{*}$ obtained in Proposition 2.2.

\section{Bifurcation analysis and possible scenarios}

\subsection{Bifurcation and global analysis}

As seen in Section 2, our model has at most three market stationary equilibria, i.e., $a=0, a=1$ and $a=a^{*}$ in (2.8), whose stability depends on the considered parameter configurations. We report the possible dynamic scenarios generated by (2.9) on varying $\Delta$ in Figures $1-6$ and some possible frameworks on varying $c_{y}$ in Figures 7-9. In particular, in all the figures below we fix the parameters as follows: $c_{x}=0.9, w_{x}=0.2, w_{y}=2, \beta=0.1, \mu=6$. Moreover, in Figures 1 and 2 we have $c_{y}=0.3$, in Figures $3-6$ we have $c_{y}=0.15$, and in all of them $\Delta$ assumes various values in $(0,0.9)$. In Figures $7-9 c_{y}$ varies in $(0,1)$ and $\Delta$ is respectively set equal to $0.6,0.7$ and 0.85 .

As concerns the scenario reproduced in Figures 1 and 2, on varying $\Delta \in(0,0.9)$ we notice, before a classical period-doubling cascade leading from the stable equilibrium $a=a^{*}$ to chaos, the occurrence of a transcritical bifurcation for the map $f$ at $a=a^{*}=1$ for $\Delta=0.131$, which causes the loss of stability of $a=1$ in 
favor of $a=a^{*}$, as soon as it enters the interval $(0,1)$. We recall that at a transcritical bifurcation a stable and an unstable equilibria merge and subsequently the stable equilibrium becomes unstable and vice versa. This is indeed what happens to $a=1$ and $a=a^{*}$ : namely, for $\Delta$ in a left neighborhood of 0.131, $a=1$ is stable and $a^{*} \notin(0,1)$ is unstable (see Figure 2 (a) for $\Delta=0.1$ ), while, for $\Delta$ in a right neighborhood of $0.131, a=1$ becomes unstable and $a^{*}$ gains stability (see Figure 2 (b) for $\Delta=0.6$, where the map $f$ is no more monotone and the convergence to $a=a^{*}$ is oscillatory), entering also the interval $(0,1)$. We observe that the stability of $a=1$ for $\Delta \in(0,0.131)$ leads to the extinction of the agents of group $\beta$, while the stability of $a=a^{*}$ for $\Delta \in(0.131,0.711)$ determines a regime characterized by the coexistence between the two groups of agents. At $\Delta=0.711, a^{*}$ becomes unstable via a period-doubling bifurcation and a stable period-two cycle emerges (see Figure 2 (c) for $\Delta=0.8$ ), followed by a cascade of flip bifurcations leading to the emergence of a one-piece chaotic attractor (see Figure 2 (d) for $\Delta=0.89$ ).

We notice that it is easy to find parameter values which generate the scenario symmetric to Figure 2 (a) with respect to the 45-degree line, with the graph of $f$ totally lying below it, and thus leading to the extinction of the agents of group $\alpha$. A possible such choice is given by $c_{x}=0.3, w_{x}=0.2, c_{y}=0.9, w_{y}=$ $2, \beta=0.1, \mu=6$ and $\Delta=0.1$, in which the values for $c_{x}$ and $c_{y}$ are inverted with respect to Figure 2. On the other hand, since $f^{\prime}\left(a^{*}\right)<1$ is always fulfilled (see the proof of Proposition 2.2), it is not possible to obtain for $f$ the scenarios symmetric to Figure 2 (b)-(d) with respect to the 45 -degree line.

We state in a formal manner the results about the transcritical and the flip bifurcations, respectively, in Propositions 3.1 and 3.2 below. We stress that the latter could be simply proven using Corollary 2.1. However, for the sake of symmetry, we prefer to employ the conditions in [13] to show the occurrence of both the transcritical and the flip bifurcations in the present scenario, in which $\Delta$ varies, rather than using this kind of argument here just for the transcritical bifurcation and in the framework considered in Figures 8 and 9, where $c_{y}$ varies, just for the flip bifurcation. Indeed, we remark that the occurrence of the 


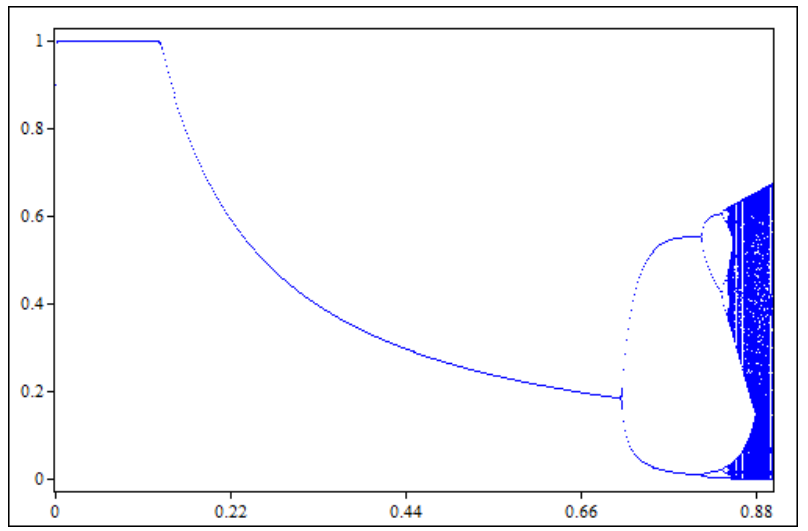

Figure 1: The bifurcation diagram of $f$ for $c_{y}=0.3$ and $\Delta \in(0,0.9)$.

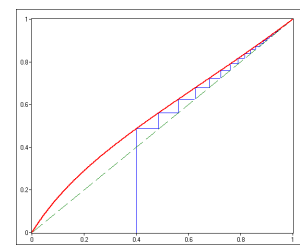

(a)

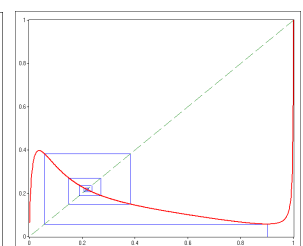

(b)

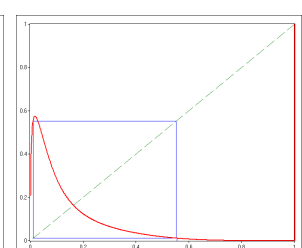

(c)

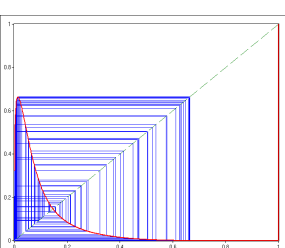

(d)

Figure 2: The graph of the first iterate of $f$ for $c_{y}=0.3$, and $\Delta=0.1, a(0)=0.4$ in (a), $\Delta=0.6, a(0)=0.9$ in (b), $\Delta=0.8, a(0)=0.555$ in (c), $\Delta=0.89, a(0)=0.6$ in (d). 
transcritical and period-doubling/period-halving bifurcations we find in Figures 3 and 7-9 could be proven as done in Propositions 3.1 and 3.2. However, in order not to overburden the paper, we chose to omit such results. In particular we notice that, although globally the case of the period-doubling bifurcations in Figures 3 and 9 is different from the other ones, since they are followed by a onepiece chaotic attractor rather than by a period-two cycle, locally the behavior of the system is the same as the one considered in Proposition 3.2 and illustrated in Figure 1.

Proposition 3.1. For the map $f=f(a ; \Delta)$ in (2.11) a transcritical bifurcation occurs at $\tilde{a}=1$ for $\widetilde{\Delta}=0.131$.

Proof. According to [13, page 507], for the occurrence of a transcritical bifurcation at a point $a=\tilde{a}$ for a certain $\Delta=\widetilde{\Delta}$ we just have to check the following conditions:

$$
\begin{array}{ll}
f(\tilde{a} ; \widetilde{\Delta})=\tilde{a}, \quad & \frac{\partial f}{\partial a}(\tilde{a} ; \widetilde{\Delta})=1, \quad \frac{\partial f}{\partial \Delta}(\tilde{a} ; \widetilde{\Delta})=0, \\
\frac{\partial^{2} f}{\partial a \partial \Delta}(\tilde{a} ; \widetilde{\Delta}) \neq 0, & \frac{\partial^{2} f}{\partial a^{2}}(\tilde{a} ; \widetilde{\Delta}) \neq 0 .
\end{array}
$$

Direct (software-assisted) computations show that the above conditions are satisfied at $\tilde{a}=1$ for $\widetilde{\Delta}=0.131$. In particular, it holds that $\frac{\partial^{2} f}{\partial a \partial \Delta}(\tilde{a} ; \widetilde{\Delta})=3.448$ and $\frac{\partial^{2} f}{\partial a^{2}}(\tilde{a} ; \widetilde{\Delta})=0.902$. This completes the proof.

Proposition 3.2. For the map $f=f(a ; \Delta)$ in (2.11) a period-doubling bifurcation occurs at $\widehat{a}=a^{*}=0.187$ for $\widehat{\Delta}=0.711$.

Proof. According to [13, page 516], for the occurrence of a period-doubling bifurcation at a point $a=\widehat{a}$ for a certain $\Delta=\widehat{\Delta}$ we have to check the following conditions:

$$
\begin{array}{ll}
f(\widehat{a} ; \widehat{\Delta})=\widehat{a}, \quad \frac{\partial f}{\partial a}(\widehat{a} ; \widehat{\Delta})=-1, \quad \frac{\partial f^{2}}{\partial \Delta}(\widehat{a} ; \widehat{\Delta})=0, \\
\frac{\partial^{2} f^{2}}{\partial a \partial \Delta}(\widehat{a} ; \widehat{\Delta}) \neq 0, \quad \frac{\partial^{2} f^{2}}{\partial a^{2}}(\widehat{a} ; \widehat{\Delta})=0, \quad \frac{\partial^{3} f^{2}}{\partial a^{3}}(\widehat{a} ; \widehat{\Delta}) \neq 0 .
\end{array}
$$

Direct (software-assisted) computations show that the above conditions are satisfied at $\widehat{a}=0.187$ for $\widehat{\Delta}=0.711$. In particular, it holds that $\frac{\partial^{2} f^{2}}{\partial a \partial \Delta}(\widehat{a} ; \widehat{\Delta})=6.895$ and $\frac{\partial^{3} f^{2}}{\partial a^{3}}(\widehat{a} ; \widehat{\Delta})=-20.492$. This completes the proof. 


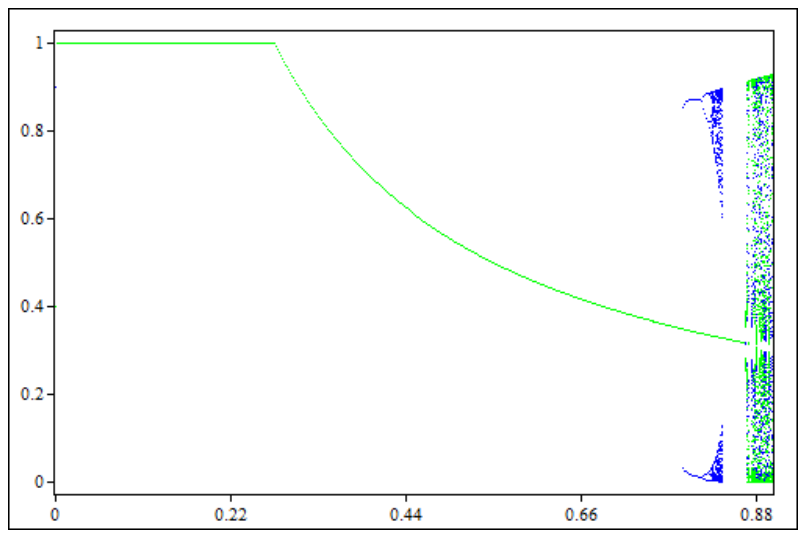

Figure 3: The bifurcation diagram of $f$ for $c_{y}=0.15$ and $\Delta \in(0,0.9)$, with initial conditions $a(0)=0.4$ for the green points and $a(0)=0.9$ for the blue points.

In Figures 3-6 we consider the same parameter configuration employed to draw Figures 1 and 2, except for the value of $c_{y}$, which from $c_{y}=0.3$ becomes $c_{y}=0.15$. In such new framework, in addition to the transcritical bifurcation occurring at $a=a^{*}=1$ for $\Delta=0.275$, in correspondence to which $a=1$ becomes unstable in favor of $a=a^{*}$, which enters the interval $(0,1)$, and to a period-doubling cascade to chaos, starting with the flip bifurcation of $a=a^{*}=$ 0.317 for $\Delta=0.867$, we also observe multistability phenomena characterized by the coexistence between the stable equilibrium $a=a^{*}$ with an external, periodic or chaotic, two-piece attractor. More precisely, the external attractor emerges for $\Delta=0.788$ as a period-two cycle, which then undergoes a cascade of period-doubling bifurcations, leading to a two-piece chaotic attractor, which disappears for $\Delta=0.840$, as shown in Figure 3. For $\Delta \in(0.840,0.867)$ the unique attractor is given by $a=a^{*}$, which, after undergoing a flip bifurcation for $\Delta=0.867$, generates a one-piece chaotic attractor (see Figure 3).

In order to understand the emergence and the disappearance of the external attractor existing for $\Delta \in(0.788,0.840)$, we report the graph of the second iterate of $f$ for values of $\Delta$ in a neighborhood of 0.788 in Figure 4 , and for values of $\Delta$ in a neighborhood of 0.840 in Figure 5, respectively.

In particular, in Figure 4 (a), for $\Delta=0.77$, the only nontrivial steady state, 


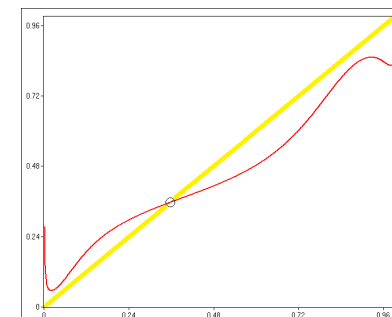

(a)

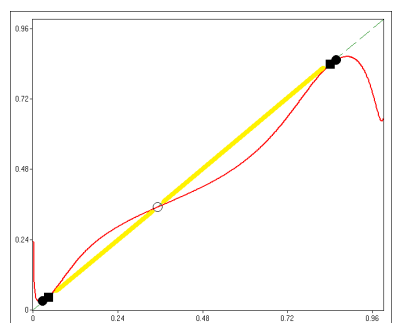

(b)

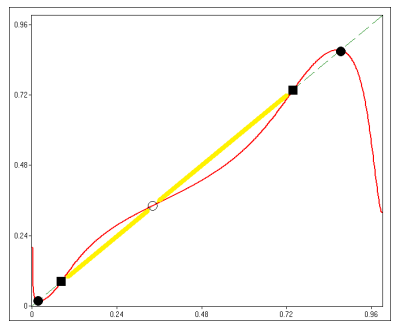

(c)

Figure 4: The graph of the second iterate of $f$ for $\Delta=0.77$ in (a), $\Delta=0.788$ in (b) and $\Delta=0.8$ in (c). We highlight in yellow the immediate basin of attraction of $a=a^{*}$.

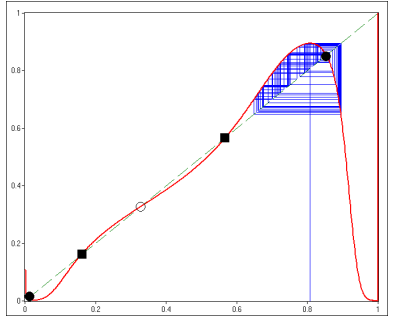

(a)

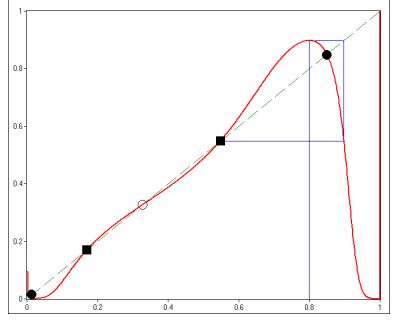

(b)

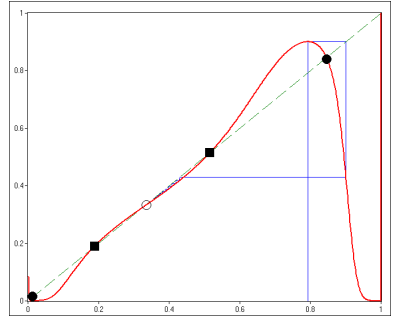

(c)

Figure 5: The graph of the second iterate of $f$ for $\Delta=0.835$ in (a), $\Delta=0.840$ in (b) and $\Delta=0.845$ in (c). We also show the forward iterates for $f^{2}$ of its maximum point $a(0)=0.807$ in (a), of $a(0)=0.799$ in (b) and of $a(0)=0.792$ in (c), respectively. 
denoted by a circle, is given by $a=a^{*}$. It is stable and its basin of attraction, colored in yellow in the same picture, coincides with $(0,1)$. In Figure $4(\mathrm{~b})$, for $\Delta=0.788$, two period-two cycles of $f$, one of which stable and the other one unstable, emerge through a double fold bifurcation of $f^{2}$ at $a=0.04$ and at $a=0.839$. We state in a formal manner the corresponding result in the next proposition.

Proposition 3.3. Given the map $f=f(a ; \Delta)$ in (2.11), a fold bifurcation for $f^{2}$ simultaneously occurs at $\breve{a_{1}}=0.04$ and at $\breve{a_{2}}=0.839$ for $\breve{\Delta}=0.788$.

Proof. According to [13, page 503], for the occurrence of a fold bifurcation of $f^{2}$ at a point $a=\breve{a}$ for a certain $\Delta=\breve{\Delta}$ we have to check the following conditions:

$$
\begin{aligned}
& f^{2}(\breve{a} ; \breve{\Delta})=\breve{a}, \quad \frac{\partial f^{2}}{\partial a}(\breve{a} ; \breve{\Delta})=1, \quad \frac{\partial f^{2}}{\partial \Delta}(\breve{a} ; \breve{\Delta}) \neq 0, \\
& \frac{\partial^{2} f^{2}}{\partial a^{2}}(\breve{a} ; \breve{\Delta}) \neq 0 .
\end{aligned}
$$

Direct (software-assisted) computations show that the above conditions are satisfied for $\breve{\Delta}=0.788$ at both $\breve{a_{1}}=0.04$ and $\breve{a_{2}}=0.839$. In particular, it holds that $\frac{\partial f^{2}}{\partial \Delta}\left(\breve{a_{1}} ; \breve{\Delta}\right)=-1.373, \frac{\partial f^{2}}{\partial \Delta}\left(\breve{a_{2}} ; \breve{\Delta}\right)=2.662, \frac{\partial^{2} f^{2}}{\partial a^{2}}\left(\breve{a_{1}} ; \breve{\Delta}\right)=31.188$ and $\frac{\partial^{2} f^{2}}{\partial a^{2}}\left(\breve{a_{2}} ; \breve{\Delta}\right)=-15.942$. This completes the proof.

We stress that the unstable period-two cycle bounds the immediate basin of attraction of $a=a^{*}$, which is still colored in yellow in both Figures 4 (b) and (c). In the latter, for $\Delta=0.8$, we denote by squares the unstable period-two cycle, whose values are given by $a=0.084$ and by $a=0.741$, and we denote by dots the stable period-two cycle, whose values are given by $a=0.016$ and by $a=0.872$. The stable period-two cycle, which with the increase of $\Delta$ undergoes a cascade of period-doubling bifurcations, leading to a two-piece chaotic attractor, is the one we see in Figure 3.

In Figure 5 we report the forward iterates for $f^{2}$ of its maximum point for $\Delta=0.835$ in (a), $\Delta=0.840$ in (b) and $\Delta=0.845$ in (c), respectively. We notice that for $\Delta=0.835$ the forward iterates for $f^{2}$ of its maximum point do not belong to the immediate basin of attraction of $a=a^{*}$, bounded by the unstable period-two cycle, while for $\Delta=0.840$ the second iterate for $f^{2}$ of its 
maximum point coincides with the larger element of the unstable period-two cycle, and for $\Delta=0.845$ the second iterate for $f^{2}$ of its maximum point falls within the immediate basin of attraction of $a=a^{*}$. Indeed, with the increase of $\Delta$, the shape of the map $f$ changes, and in particular the maximum value of $f^{2}$ raises and falls within the component of the basin of attraction of $a=a^{*}$ which extends up to $a=1$. This explains why the external attractor disappears for $\Delta=0.840$ : namely, its two components are internally bounded by the unstable period-two cycle, while they are externally bounded by the maximum and minimum values assumed by the map $f$.

Similarly, in Figure 6, in view of explaining the emergence of the one-piece chaotic attractor existing for $\Delta \in(0.867,0.9)$, we report the graph of $f^{2}$ for values of $\Delta$ in a neighborhood of 0.867. In particular, in Figure 6 (a) for $\Delta=0.846$ the unstable period-two cycle, denoted by squares, still exists and disappears for $\Delta=0.867$ due to a reverse pitchfork bifurcation of $f^{2}$ at $a=a^{*}$, just when $f^{\prime}\left(a^{*}\right)=-1$ and the map $f$ undergoes a flip bifurcation (see Figure $6(\mathrm{~b})$ ). We state in a formal manner the result on the pitchfork bifurcation in Proposition 3.4 below. In Figure 6 (c), for $\Delta=0.890$, the unstable period-two cycle does not exist anymore. The disappearance of the unstable period-two cycle in correspondence to the flip bifurcation implies that the chaotic attractor following it is composed just by one piece: indeed, as explained above, the two components of the external attractor existing for $\Delta \in(0.788,0.840)$ were internally bounded by the unstable period-two cycle, whose values move closer and closer to $a=a^{*}$ with the increase of $\Delta$, until they collide with $a^{*}=0.317$ for $\Delta=0.867$. Hence, for $\Delta>0.867$ even the points lying in a neighborhood of $a=a^{*}$ belong to the one-piece chaotic attractor, as the immediate basin of attraction of $a^{*}$ has collapsed.

Proposition 3.4. Given the map $f=f(a ; \Delta)$ in (2.11), a pitchfork bifurcation for $f^{2}$ occurs at $\bar{a}=a^{*}=0.317$ for $\bar{\Delta}=0.867$.

Proof. According to [13, page 511], for the occurrence of a pitchfork bifurcation of $f^{2}$ at a point $a=\bar{a}$ for a certain $\Delta=\bar{\Delta}$ we have to check the following 


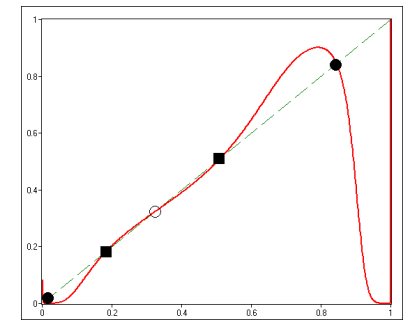

(a)

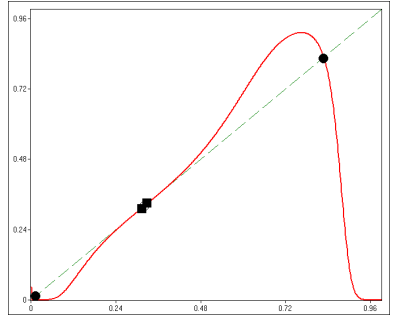

(b)

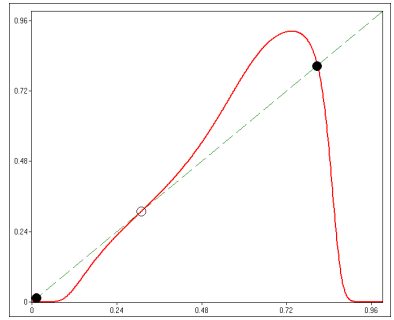

(c)

Figure 6: The graph of the second iterate of $f$ for $\Delta=0.846$ in (a), $\Delta=0.867$ in (b) and $\Delta=0.890$ in (c).

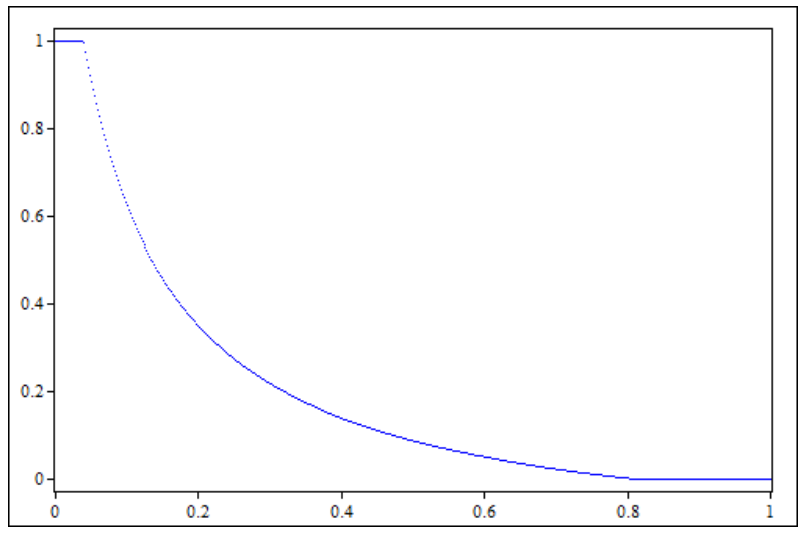

Figure 7: The bifurcation diagram of $f$ for $\Delta=0.6$ and $c_{y} \in(0,1)$.

conditions:

$$
\begin{array}{ll}
f^{2}(\bar{a} ; \bar{\Delta})=\bar{a}, \quad & \frac{\partial f^{2}}{\partial a}(\bar{a} ; \bar{\Delta})=1, \quad \frac{\partial f^{2}}{\partial \Delta}(\bar{a} ; \bar{\Delta})=0, \\
\frac{\partial^{2} f^{2}}{\partial a^{2}}(\bar{a} ; \bar{\Delta})=0, & \frac{\partial^{2} f^{2}}{\partial a \partial \Delta}(\bar{a} ; \bar{\Delta}) \neq 0, \quad \frac{\partial^{3} f^{2}}{\partial a^{3}}(\bar{a} ; \bar{\Delta}) \neq 0 .
\end{array}
$$

Direct (software-assisted) computations show that the above conditions are satisfied at $\bar{a}=a^{*}=0.317$ for $\bar{\Delta}=0.867$. In particular, it holds that $\frac{\partial^{2} f^{2}}{\partial a \partial \Delta}(\bar{a} ; \bar{\Delta})=$ 6.758 and $\frac{\partial^{3} f^{2}}{\partial a^{3}}(\bar{a} ; \bar{\Delta})=34.160$. This completes the proof.

Looking at Figures 1-6, we observe that decreasing the value of $c_{y}$ from $c_{y}=0.3$ in Figures 1 and 2 to $c_{y}=0.15$ in Figures $3-6$ generates more complex dynamics. Let us now investigate in greater detail the role of the parameter $c_{y}$ in Figures 7-9, where we report the bifurcation diagrams for $c_{y} \in(0,1)$, and $\Delta=0.6$ in Figure $7, \Delta=0.7$ in Figure 8 and $\Delta=0.85$ in Figure 9 , respectively. 


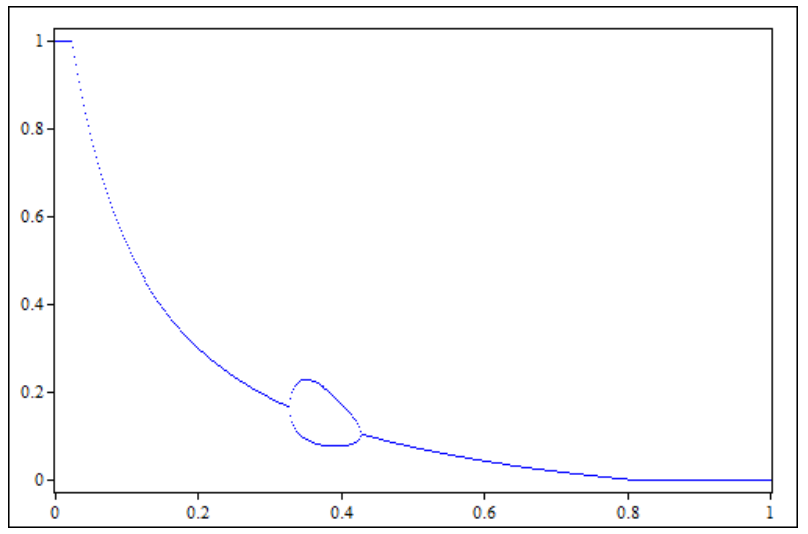

Figure 8: The bifurcation diagram of $f$ for $\Delta=0.7$ and $c_{y} \in(0,1)$.

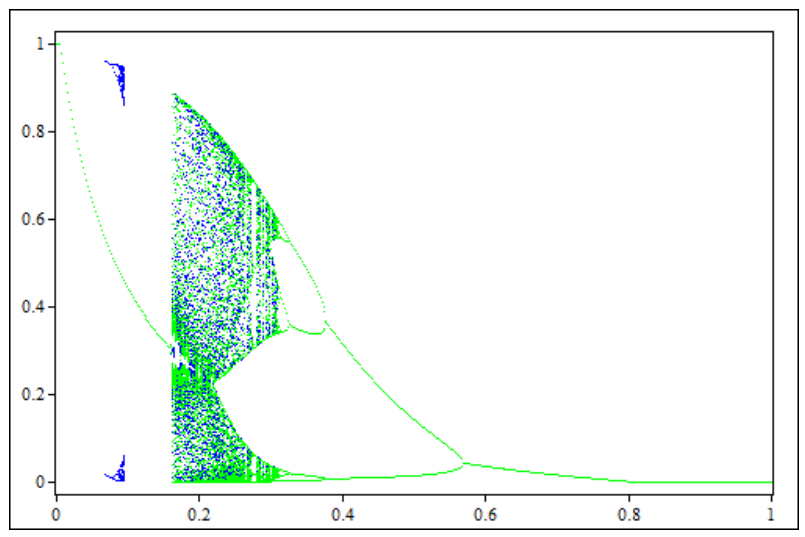

Figure 9: The bifurcation diagram of $f$ for $\Delta=0.85$ and $c_{y} \in(0,1)$, with initial conditions $a(0)=0.4$ for the green points and $a(0)=0.95$ for the blue points. 
We notice that increasing the value of $\Delta$ generally complicates the dynamic behavior, while for $c_{y}$ we usually find a mixed behavior, characterized by the presence of two stability threshold values.

More precisely, in Figure 7 the equilibrium $a=a^{*}$ is asymptotically stable for every value of $c_{y}$ for which $a^{*} \in(0,1)$, and we observe two transcritical bifurcations when $a=a^{*}$ enters and leaves the interval $(0,1)$, respectively for $c_{y}=0.038$ and $c_{y}=0.81$.

In Figure 8, in addition to the same two transcritical bifurcations as in Figure 7 , occurring now for $c_{y}=0.022$ and $c_{y}=0.81$, we find a "bubble" (see $[2,3,4,5,6])$. Indeed, $a=a^{*}$ is stable for $c_{y} \in(0.022,0.326) \cup(0.428,0.81)$, while for $c_{y} \in(0.326,0.428)$ we have a period-two cycle, which starts with a period-doubling bifurcation for $c_{y}=0.326$ and ends with a period-halving bifurcation for $c_{y}=0.428$. We stress that such values for $c_{y}$ are consistent with the value of $\mu^{*}$ derived in Proposition 2.2: namely, when inserting $c_{y}=0.326$ or $c_{y}=0.428$ in that expression, together with the other parameter values considered in this scenario, we obtain $\mu^{*}=6$, which coincides with the value for $\mu$ we are taking into account. The same remark applies also to the period-doubling and period-halving bifurcations we find in Figure 9.

In Figure 9, we still have the two transcritical bifurcations, occurring now for $c_{y}=0.005$ and $c_{y}=0.81$, as well as the period-doubling and period-halving bifurcations bounding the (local) stability regions, occurring now for $c_{y}=0.160$ and $c_{y}=0.568$, respectively. However, for $c_{y} \in(0.160,0.568)$, we do not observe just a period-two cycle like in Figure 8, but much more complex dynamics, characterized by the initial presence of a one-piece chaotic attractor, which then undergoes a cascade of period-halving bifurcations, until a period-two cycle. Moreover, for $c_{y} \in(0.065,0.094)$, we find a multistability phenomenon analogous to the one detected in Figure 3 when increasing the value of $\Delta$ and that could be explained with the same arguments employed in Figures 4 and 5. However, differently from that framework, we do not end up anymore with a chaotic regime, but rather with a stable steady state. Indeed, the system recovers stability via the period-halving bifurcation occurring for $c_{y}=0.568$, 
which makes $a=a^{*}$ become stable, and then $a=a^{*}$ loses its stability in favor of $a=0$ through the transcritical bifurcation occurring for $c_{y}=0.81$. Hence, in such scenario, the final outcome is characterized not only by stability, but also by population homogeneity, as the agents of group $\alpha$ eventually disappear from the system and only the agents of group $\beta$ survive.

\subsection{Economic scenarios}

We give an economic interpretation of two main scenarios we found, i.e., those in Figure 2 (a) and (c). Indeed, the former leads to the extinction of one of the two groups of agents, while in the latter we found an oscillatory, non-converging behavior, characterized by an alternating predominance of one of the two groups of agents. For simplicity, we focus on the simplest oscillatory behavior, that is, a period-two cycle.

We stress that in Figure 2 (a) the agents of group $\beta$ become extinct. Analogous arguments allow to explain the extinction of the agents of group $\alpha$, when considering, e.g., the parameter values reported in Subsection 3.1 which generate the scenario symmetric to Figure 2 (a) with respect to the 45-degree line.

Focusing on Figure 2 (a), for instance taking $a(0)=0.4$, it holds that $K_{\beta}<K_{\alpha}$ and thus we expect an increase in the share of the agents of group $\alpha$, i.e., for the next period, a larger value for $a$ as, according to (2.10), the relative position between $K_{\alpha}$ and $K_{\beta}$ determines the population shares. We foresee this will produce an increase in the aggregate demand of commodity $x$, agents of group $\alpha$ have a stronger preference for, and that in turn this leads to a higher value for $p_{x}$, producing a decrease in the relative price $p=p_{y} / p_{x}$, that indeed we do observe. We stress that, although it is not possible to observe aggregate demand, we may infer its behavior from the shares and we can find a confirmation of it by looking at the value of $p$. The market closes and the optimal consumption quantities for the two goods are determined, which allow to compute $K_{\alpha}$ and $K_{\beta}$ and consequently the population shares. In particular, due to the decrease in $p$, the value of $x_{\alpha}^{*}$ decreases more than $x_{\beta}^{*}$ and, since $c_{x}$ is larger than $c_{y}$, the value of $K_{\alpha}$ decreases, while the value of $K_{\beta}$ increases, so that the distance 
between $K_{\beta}$ and $K_{\alpha}$ is reduced, but the ordering between them is maintained. Then, the same process repeats again, leading to larger and larger values for $a$ and to the eventual extinction of the agents of group $\beta$.

In regard to Figure 2 (c), let us start from an initial condition close to the smallest between the two elements of the period-two cycle, taking for instance $a(0)=0.05$. In such framework, the agents of type $\alpha$, with their stronger preference for commodity $x$, are few and thus we expect that the aggregate demand for that good is low. Indeed, we do observe a high value for the relative price $p$. The optimal consumption quantities for the two goods are determined and, since $p$ is high, the value of $x_{\alpha}^{*}$ is high, too. Due to the fact that $c_{x}$ is larger than $c_{y}$, we find that $K_{\alpha}>K_{\beta}$, so that the share of agents of type $\alpha$ raises and approaches $a=0.47$. We then expect that the aggregate demand for commodity $x$ is higher than before. Indeed, we observe a lower value for the relative price $p$. The optimal consumption quantities for the two goods are determined and, since $p$ is lower now, the value of $x_{\alpha}^{*}$ decreases. As $c_{x}$ is still larger than $c_{y}$, we find that $K_{\alpha}<K_{\beta}$, so that the share of agents of type $\alpha$ decreases and is again close to $a=0.05$, giving rise to a period-two cycle.

\section{Conclusion}

In the present paper, we proposed a discretization of the exchange economy evolutionary continuous-time model with agents heterogeneous in the structure of preferences introduced by Chang and Stauber in [1], in which the share updating mechanism depends in a monotone manner on the goods' consumption, described in terms of the calorie intakes. In particular, following [11, 12], we considered an exponential discrete replicator rule to describe the evolutionary mechanism. For our system, we investigated the existence and local stability of the steady states and we performed a qualitative bifurcation analysis in order to illustrate the possible scenarios, which turn out to be much richer than in the discrete-time framework. Indeed, in addition to highlighting the presence of transcritical and flip bifurcations, we detected multistability phenomena involving a steady state and a periodic or chaotic attractor surrounding it. We 
then gave an economic interpretation of the main scenarios we found.

As concerns possible future study directions, we are going to build a discretization also of the continuous-time model considered in [7], where, in a context similar to that proposed in [1], the share updating mechanism is assumed to depend in a non-monotone manner on the goods' consumption, still described in terms of the calorie intakes. Moreover, the latter framework could be modified to represent the fashion cycle. In such case, we would still deal with a bell-shaped map, describing, rather than the relationship between calorie intake and population growth rate, the link between consumption and imitative behavior, below the saturation level, and between consumption and snob behavior, above such level. In order to interpret the fashion cycle, and in particular its multistability phenomena, we need to identify (at least) two lifestyles, de-

scribed by different preference structures; for each lifestyle we would introduce an attractivity degree, which depends in a nonlinear bell-shaped manner on the consumption of the representative agent belonging to the population share who adopts that particular preference structure. Then, the two attractivities would jointly determine the population switching mechanism between the different lifestyles.

This research did not receive any specific grant from funding agencies in the public, commercial, or not-for-profit sectors.

\section{References}

[1] J. Chang and R. Stauber, Evolution of preferences in an exchange economy, Econ. Lett. 103 (2009), pp. 131-134.

[2] B.-S. Du, Point bifurcations for some one-parameter families of interval maps, Bull. Inst. Math. Acad. Sin. 21 (1993), pp. 187-202.

[3] B.-S. Du, Point bifurcations and bubbles for some one-parameter families of quadratic polynomials, Bull. Inst. Math. Acad. Sin. 25 (1997), pp. 1-9. 
[4] C.H. Hommes, Adaptive learning and roads to chaos: The case of the cobweb, Econ. Lett. 36 (1991), pp. 127-132.

[5] C.H. Hommes, Dynamics of the cobweb model with adaptive expectations and nonlinear supply and demand, J. Econ. Behav. Org. 24 (1994), pp. 315-335.

[6] M.-C. Li, Point bifurcations and bubbles for a cubic family, J. Differ. Equations Appl. 9 (2003), pp. 553-558.

[7] A. Naimzada and M. Pireddu, Endogenous evolution of heterogeneous consumers preferences: Multistability and coexistence between groups, Econ. Lett. 142 (2016), pp. 22-26.

[8] A. Naimzada and M. Pireddu, Strong coexistence for a model with endogenous evolution of heterogeneous agents, (submitted).

[9] A. Naimzada and M. Pireddu, Complex dynamics in an evolutionary general equilibrium model, (submitted).

[10] G. Ponthiere, Existence and stability of overconsumption equilibria, Econ. Modell. 28 (2011), pp. 74-90.

[11] W.H. Sandholm, Population Games and Evolutionary Dynamics, MIT Press, Cambridge, 2010.

[12] P.D. Taylor and L.B. Jonker, Evolutionarily stable strategies and game dynamics, Math. Biosci. 40 (1978), pp. 145-156.

[13] S. Wiggins, Introduction to Applied Nonlinear Dynamical Systems and Chaos, Texts in Applied Mathematics, 2nd ed., Springer, New York, 2003. 